apuntesuniversitarios.upeu.edu.pe

Apuntes Universitarios, 2021: 11(1), enero-marzo ISSN: 2304-0335 DOI: https://doi.org/10.17162/au.v11i1.542

\title{
Efecto de la enseñanza de ajedrez en las habilidades matemáticas, de atención y concentración en niños en edad escolar de la amazonía peruana
}

\section{Effect of chess teaching on mathematical, attention and concentration abilities in school- aged children of the Peruvian Amazon}

\author{
Jorge Santiago Garate-Quispe, ${ }^{1}$ Noemí Liz Quispe-Aviles, ${ }^{2}$ Lucero Aymachoque-Aslla, ${ }^{3}$ \\ Marilu Farfan Latorre ${ }^{4}$ y Oliver Surco-Huacachi ${ }^{5}$ \\ Universidad Nacional Amazónica de Madre de Dios, Perú ${ }^{12345}$ \\ Orcid ID: https://orcid.org/0000-0002-7494-2274 \\ Orcid ID: https://orcid.org/0000-0002-4038-8228² \\ Orcid ID: https://orcid.org/0000-0002-2702-0138 3 \\ Orcid ID: https://orcid.org/0000-0002-6061-996X ${ }^{4}$ \\ Orcid ID: https://orcid.org/0000-0001-9588-1469
}

Recibido: 08 de junio de 2020

Aceptado: 15 de setiembre 2020

\section{Resumen}

El presente estudio analiza la influencia del aprendizaje de ajedrez sobre las habilidades en razonamiento matemático, atención y concentración en niños en edad escolar de la Amazonia peruana. Se seleccionaron estudiantes de primer grado de secundaria de dos instituciones educativas de la ciudad de Puerto Maldonado, pública y privada. El diseño del estudio fue cuasiexperimental, con evaluaciones pretest-postest y un grupo control. Cada grupo tenía entre 18 a 30 estudiantes y al grupo experimental se le brindó clases de ajedrez dos veces por semana durante tres meses. Para analizar el efecto de la intervención se utilizó un Modelo Linear Generalizado. En las dos habilidades evaluadas los resultados del postest del grupo experimental fueron superiores al grupo control. Se encontró un efecto significativo de la aplicación del ajedrez deportivo en el desarrollo de habilidades en razonamiento matemático y atención y concentración $(P<0.001)$. En la institución pública el efecto del programa en razonamiento lógico matemático fue superior en los varones que en las mujeres $(P<0.05)$. Nuestros resultados aportan información relevante para que las instituciones educativas puedan desarrollar estrategias para promover el ajedrez como una herramienta y estrategia pedagógica. Se concluye que la implementación de un programa ajedrez influye en el desarrollo de habilidades intelectuales en escolares de la amazonia peruana.

${ }^{1}$ Correspondencia al autor:

E-mail:jgarate@unamad.edu.pe 
Palabras clave: Ajedrez, desarrollo cognitivo, matemáticas, Amazonía peruana, infancia, aprendizaje, concentración

\begin{abstract}
The study aimed to analyze the influence of learning chess on mathematical, attention, and concentration abilities in school-aged children of two Schools in Peruvian Amazon. First-grade secondary school students were selected from two schools in Puerto Maldonado city, public and private. The design of the study was quasi-experimental, pretest-posttest evaluations and a control group. Each group had between 18-30 students, and the experimental group was given chess classes twice a week for three months. To analyze the effect of the intervention a Generalized Linear Model was used. In the two abilities evaluated, the posttest results of the experimental group were higher than the control group. A significant effect of the application of sports chess was found in the development of abilities in mathematical reasoning, attention, and concentration $(P<0.001)$. In the public school, the effect of the program on mathematical reasoning was higher in boys than in girls $(P<0.05)$. Our results provide relevant information for schools to develop strategies to promote chess as a teaching strategy. We concluded that the implementation of a chess program influences the development of the intellectual abilities in school-aged children of Peruvian Amazon.
\end{abstract}

Keywords: Chess, cognitive development, mathematics, Peruvian Amazon, childhood, learning, concentration

\title{
Introducción
}

Como deporte, el ajedrez es reconocido como un paradigma en la investigación cognitiva (Aciego, García, y Betancort, 2016); asimismo, viene siendo utilizado en la educación básica para fortalecer el desarrollo cognitivo, afectivo y psicomotor de escolares a mediano y largo plazo (Avalos et al., 2019; Nakao, 2019; Ramos, Arán-Filippetti, y Krumm, 2017). Por estas razones, el ajedrez, además de un deporte, ciencia y arte, es también reconocido como una excelente herramienta educativa (Fernández y Sánchez, 2011); esto señala que la estrategia intrínseca al ajedrez implicaría un proceso de investigación científica (Lara-Piña, et al., 2018). Según Avalos et al. (2019), a nivel mundial más de 30 millones de escolares participan semanalmente en programas educativos que utilizan el ajedrez como herramienta, el 10\% estos corresponden a América.

En este contexto, se han desarrollado varios estudios a nivel mundial para demostrar o refutar los beneficios de la práctica del ajedrez en el rendimiento académico de escolares (Fernández et al., 2018; Jankovic y Novak, 2019; Kovacic, 2012; Nakao, 2019; Poston y Vandenkieboom, 2019; Ramos et al., 2017; Rosholm et al., 2017), especialmente con respecto a las habilidades matemáticas (Trinchero, 2013; Trinchero y Sala, 2016). Según Jankovic y Novak 
(2019), esta tendencia se debe a que el ajedrez influye fisiológicamente en la corteza del cerebro y de esta manera puede mejorar las habilidades en matemáticas. Poston y Vandenkieboom (2019) sugieren que los niños dedicados al ajedrez pueden mejorar significativamente en dichas ciencias, entre 30-50\%. Por otro lado, Ramos et al. (2017) y Gao, Chen, Wang, y Lin (2019) sugieren que la practica del ajedrez puede mejorar las funciones ejecutivas y visuales de los niños (8-12 años de edad), mientras que Kovacic (2012) reporta que la practica del ajedrez tambien puede influir en el aprendizaje de las ciencias naturales y sociales. De igual forma, se cree que la practica del ajedrez en niños en edad escolar podría disminuir el deterioro cognitivo y la depresión (Kovacic, 2012; Nakao, 2019) y evitar la discapacidad de aprendizaje e intelectual (Scholz et al., 2008; Storey, 2000).

Considerando la importancia de los programas de ajedrez en las escuelas, son escasos los estudios que evalúen la influencia de aquello en el desarrollo cognitivo de estudiantes peruanos. Reluz (2019) reportó la relación entre el ajedrez y el desarrollo de las habilidades matemáticas, mientras que otros trabajos teóricos sugieren que tal juego de estrategia puede mejorar los niveles de atención en estudiantes de primaria (García, 2018; Salinas, 2015).

En este caso, el informe de la Organización para la Cooperación y el Desarrollo Económico (OCDE), en relación al programa de evaluación internacional estudiantes (PISA) precisa que de 77 países, el Perú se ubica entre los últimos lugares, con el puntaje de 64 en matemática, lectura y ciencias (OECD, 2019). Los resultados de la evaluación PISA son modestos en todos los países de Latinoamérica (Muelle, 2020), aunque en matemáticas el Perú solo supera a Colombia, Argentina y Brasil (OECD, 2019). Además del PISA, evaluaciones nacionales como la evaluación censal de estudiantes (ECE), reportan resultados similares (MINEDU, 2018a). Un ejemplo: la región Madre de Dios, en el ECE-2018, presentó las tasas de desaprobación y retiro más altas del Perú, lo que es particularmente preocupante por la desaprobación en el nivel secundario (20\%), siendo el doble que el indicador nacional (10.3\%) (MINEDU, 2015).

Por estas razones, es esencial implementar estrategias que mejoren el desarrollo de las habilidades intelectuales, y una alternativa sería implementar en las escuelas programas de aprendizaje y entrenamiento de ajedrez como herramienta pedagógica. En este escenario, los resultados del presente estudio tienen una gran importancia. Por ello, el presente estudio se considera importante porque cubrirá un vacío de información sobre la aplicación del ajedrez deportivo para el desarrollo de habilidades intelectuales en escolares peruanos; considerando que 
no existen estudios similares en el contexto de la Amazonia peruana. Además, aunque se hayan encontrado estudios que reportan una relación significativa entre el ajedrez y el desarrollo cognitivo en estudiantes de primaria y secundaria, son escasos los estudios con antecedentes experimentales (Lara-Piña et al., 2018), y ahí radica la importancia del presente estudio. A la luz de estos antecedentes, el objetivo del estudio es analizar el efecto de un programa de ajedrez en el desarrollo de habilidades intelectuales en estudiantes de dos instituciones educativas de la Amazonia peruana (Madre de Dios).

\section{Materiales y métodos}

\section{Diseño y ámbito}

Se realizó la intervención a estudiantes de primer grado de educacion secundaria en dos instituciones educativas (IEs), una privada y otra publica, de la ciudad de Puerto Maldonado, Madre de Dios (Perú). El diseño del presente estudio es cuasi experimental, antes (pretest) y después (postest), con dos grupos (control y tratamiento). Como variable dependiente se analizó la competencia cognitiva, evaluada mediante las habilidades en: (1) razonamiento lógico matemático y (2) atención y concentración, aplicadas al iniciar el tratamiento (pre-test) y al finalizar (post-test).

\section{Sujetos de estudio}

La muestra ha sido extraída de dos IEs de Puerto Maldonado, una de gestión pública y la otra de gestión privada, y estuvo compuesta por 95 niños (43 varones y 52 mujeres). Las edades están comprendidas entre 11 y 12 años. La selección de las IEs se realizó mediante un muestreo no probabilístico intencional, considerando las facilidades de acceso y disponibilidad. En cada IE se seleccionaron dos grupos: control y experimental; el grupo control fue el testigo y no se aplicó ningún tratamiento, mientras que al grupo experimental se le brindó clases de ajedrez. La muestra de IE pública estuvo conformada por 55 estudiantes (30 en control y 25 en experimental), mientras que en la IE privada fue de 40 estudiantes (22 en control y 18 en experimental). Por otro lado, se realizó un consentimiento informado, con el objetivo de informar a los padres de familia y profesores de las IEs sobre los objetivos del estudio y la confidencialidad de los datos personales brindados. Asimismo, se realizó un asentimiento informado a los estudiantes, menores de edad entre 11 y 12 años de edad, con la finalidad de hacerlos partícipes del estudio e informales los alcances del mismo. 


\section{Tratamiento}

Previo al tratamiento, y después del mismo, se evaluaron a ambos grupos (control y tratamiento) en sus habilidades de (1) razonamiento lógico matemático y (2) atención y concentración. Se utilizaron dos pruebas para evaluar las habilidades en matemáticas y concentración antes y después de la intervención siguiendo la metodología utilizado por Rosholm et al. (2017). Cada prueba tenía cinco ejercicios relacionados a cada habilidad. Así, la prueba de razonamiento matemático contenía reconocimiento de patrones, geometría, series numéricas y cálculos matemáticos, mientras que la prueba de atención y concentración contenía ejercicios relacionados a formas, búsqueda de repetidos, conteo y reconocimiento de patrones.

A los estudiantes del grupo experimental de ambas IE se les brindó lecciones de ajedrez deportivo 2 veces por semana, durante tres meses del 2019. Las lecciones de ajedrez en el grupo experimental fueron desarrolladas por instructores calificados del Club-Escuela de Ajedrez Botvinnik de Puerto Maldonado. Las lecciones de ajedrez fueron de nivel iniciación a básico, un método diseñado para niños (Surco-Huacachi, 2020), siguiendo la metodología propuesta por (Scholz et al., 2008). Este empieza con la familiarización con el tablero, las piezas, movimientos y valor de piezas, y ejercicios de iniciación. Para el nivel básico se desarrollaron temas de mates en una movida, aperturas y táctica.

Para determinar el rendimiento (nivel) en ajedrez en los grupos experimentales se utilizó el método estadístico Elo (Lotero y Cano, 2017). Este es un sistema establecido por la Federación Internacional de Ajedrez (FIDE) como un sistema de clasificación de jugadores de ajedrez en función a su calidad de juego (Benítez y Roca, 2018). En el estudio se calculó dos tipos de Elo: Elo-virtual y Elo-estándar. El Elo-virtual se estimó mediante el promedio obtenido con dos herramientas, el software ChessMaster v10 (partidas) y la aplicación Puzzle Chess Rush (táctica). Con el software Chessmaster, cada estudiante jugó 6 partidas (3 con blancas y 3 con negras) con el módulo de ajedrez. La aplicación Puzzle Chess Rush determinó el nivel de ajedrez en los estudiantes a través de 10 ejercicios de táctica. Para determinar el Elo estándar, se realizaron 5 partidas físicas de ajedrez y ejercicios tácticos desarrollado con el acompañamiento de los instructores de ajedrez. 


\section{Análisis estadístico}

Previo a elegir el estadístico a utilizar en el análisis del experimento, se verificó el cumplimiento de los supuestos paramétricos de normalidad y homocedasticidad. La normalidad se evaluó utilizando la prueba de Shapiro-Wilk y la homocedasticidad con el test de Levene. Los análisis se realizaron en el software PAST v. 4 (Hammer, Harper, y Ryan, 2001). En todos los análisis del estudio se utilizó un nivel de significancia de 5\%. Para comparar el Elo inicial y rendimiento inicial (habilidades) de los estudiantes se utilizaron las pruebas de T-student o ShapiroWilk, según corresponda. Los análisis se realizaron en PAST v. 4 (Hammer et al., 2001).

En el análisis del efecto de la intervención sobre los grupos se utilizó un Modelo Linear Generalizado (GLM). Los análisis se llevaron a cabo considerando como covariable a los valores del pre-test y se analizó el efecto del sexo y su interacción con la variable grupo. Se utilizó el GLM debido a que las variables analizadas no cumplen con los supuestos paramétrico (normalidad y homogeneidad, Tabla 1) y que es mejor enfoque de análisis considerando el diseño del presente estudio (Dugard y Todman, 1995; Johnson, 2016). Los análisis se realizaron con el paquete estadístico SPSS IBM Statistics (v. 26). Las figuras se realizaron utilizando los paquetes estadísticos SigmaPlot 14 y el paquete ggplot2 de R en entorno de R-Studio (R Core Team, 2017; Wickham, 2009). 
Tabla 1. Resumen del análisis de cumplimiento de supuestos paramétricos de las habilidades intelectuales (pre-test y post-test) y la fuerza ajedrecística de los estudiantes de primer grado de secundaria en dos instituciones educativas de Puerto Maldonado (Perú). Normalidad (test de Shapiro-Wilk). Homogeneidad de varianzas (test de Levene). RLM: Razonamiento Lógico Matemático. AyT: Atención y Concentración.

\begin{tabular}{|c|c|c|c|c|c|}
\hline \multirow{2}{*}{$\begin{array}{l}\text { Institución Educativa } \\
\text { Variable }\end{array}$} & \multirow[b]{2}{*}{ Grupo } & \multicolumn{2}{|c|}{ Pública } & \multicolumn{2}{|r|}{ Privada } \\
\hline & & Normalidad & $\begin{array}{l}\text { Homogeneidad de } \\
\text { varianzas }\end{array}$ & Normalidad & $\begin{array}{l}\text { Homogeneidad de } \\
\text { varianzas }\end{array}$ \\
\hline \multicolumn{6}{|c|}{ (a) Habilidad intelectual } \\
\hline RLM (Pre-test) & $\begin{array}{l}\text { Control } \\
\text { Experimental }\end{array}$ & $\begin{array}{l}P=0.054^{*} \\
P<0.001\end{array}$ & $P=0.003$ & $\begin{array}{c}P=0.130 * \\
P=0.001\end{array}$ & $P<0.001$ \\
\hline RLM (Post-test) & $\begin{array}{l}\text { Control } \\
\text { Experimental }\end{array}$ & $\begin{array}{c}P<0.001 \\
P=0.071^{*}\end{array}$ & $P=0.453^{*}$ & $\begin{array}{c}P=0.130^{*} \\
P=0.004\end{array}$ & $P=0.137^{*}$ \\
\hline AyT (Pre-test) & $\begin{array}{l}\text { Control } \\
\text { Experimental }\end{array}$ & $\begin{array}{l}P<0.001 \\
P<0.001\end{array}$ & $P<0.001$ & $\begin{array}{l}P=0.001 \\
P<0.001\end{array}$ & $P=0.002$ \\
\hline AyT (Post-test) & $\begin{array}{l}\text { Control } \\
\text { Experimental }\end{array}$ & $\begin{array}{l}P<0.001 \\
P<0.001\end{array}$ & $P=0.87^{*}$ & $\begin{array}{l}P=0.020 \\
P<0.001\end{array}$ & $P=0.031$ \\
\hline \multicolumn{6}{|c|}{ (b) Puntuación de fuerza ajedrecística (Elo) } \\
\hline $\begin{array}{l}\text { Elo virtual inicial } \\
\text { Elo virtual final }\end{array}$ & $\begin{array}{l}\text { Experimental } \\
\text { Experimental }\end{array}$ & $\begin{array}{l}P<0.172 * \\
P<0.294 *\end{array}$ & $P=0.013$ & $\begin{array}{l}P=0.013 \\
P=0.042\end{array}$ & $P=0.67^{*}$ \\
\hline $\begin{array}{l}\text { Elo estándar inicial } \\
\text { Elo estándar final }\end{array}$ & $\begin{array}{l}\text { Experimental } \\
\text { Experimental }\end{array}$ & $\begin{array}{l}P<0.172 * \\
P<0.294 *\end{array}$ & $P=0.009$ & $P=0.012$ & $P=0.022$ \\
\hline
\end{tabular}

\section{Resultados}

Los resultados del análisis comparativo entre los grupos control y experimental antes de la aplicación del tratamiento según la institución educativa (Tabla 2) sugieren que no existen diferencias significativas en el razonamiento lógico matemático, atención y concentración entre los grupos control y experimental (test de Mann-Whitney, $P>0.05$ ), lo cual establece que los grupos presentan rendimientos similares antes de aplicar el tratamiento. Lo cual es conveniente en el tipo de estudio planteado (Sotelo, Sotelo, Matalinares, Arenas Iparraguirre, y Caycho, 2014). 
Tabla 2. Resultados de la comparación del rendimiento inicial de los estudiantes entre los grupos control y experimental previo a la aplicación del tratamiento, utilizando la prueba de Mann-Whitney, en estudiantes de primer grado de secundaria en dos instituciones educativas de Puerto Maldonado (Perú). Min: Mínimo. Max: Máximo. SD: Desviación estándar. CV: Coeficiente de variación.

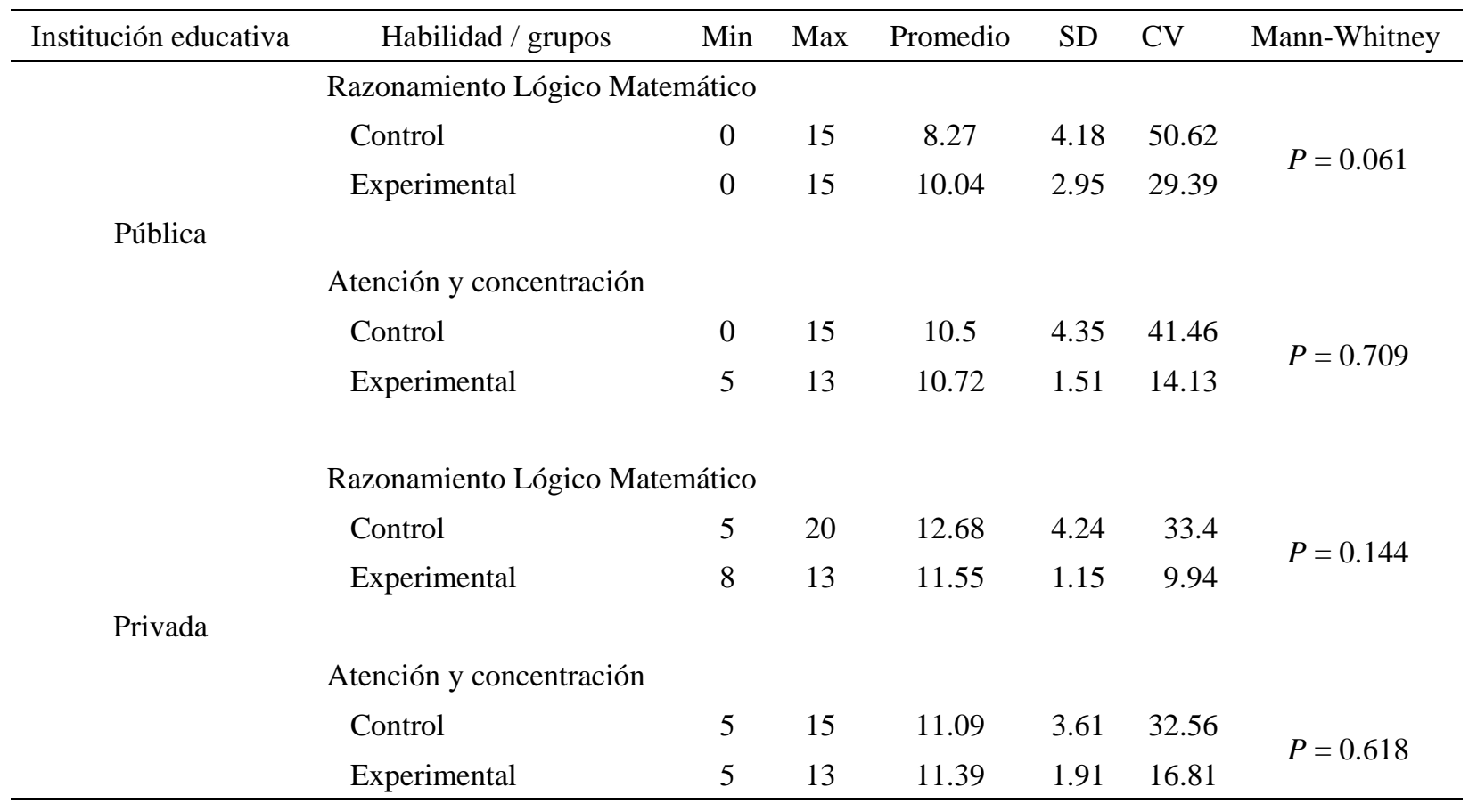

\section{Variaciones de Elo luego del tratamiento}

La comparación estadística entre los grupos, mostró un incremento significativo en el Elo virtual y estándar en los estudiantes de las IE evaluadas (U de Mann-Whitney, $P<0.05$, Tabla 2, Figura 1A, 1B y 1D), a excepción del Elo virtual en la IE privada (U de Mann-Whitney, $P=0.07$, Tabla 2, Figura 1B). El incremento promedio de los puntajes del Elo estándar de los estudiantes fue de $130 \pm 37$ puntos, superior al incremento en Elo virtual que fue de 130 \pm 103 puntos. 

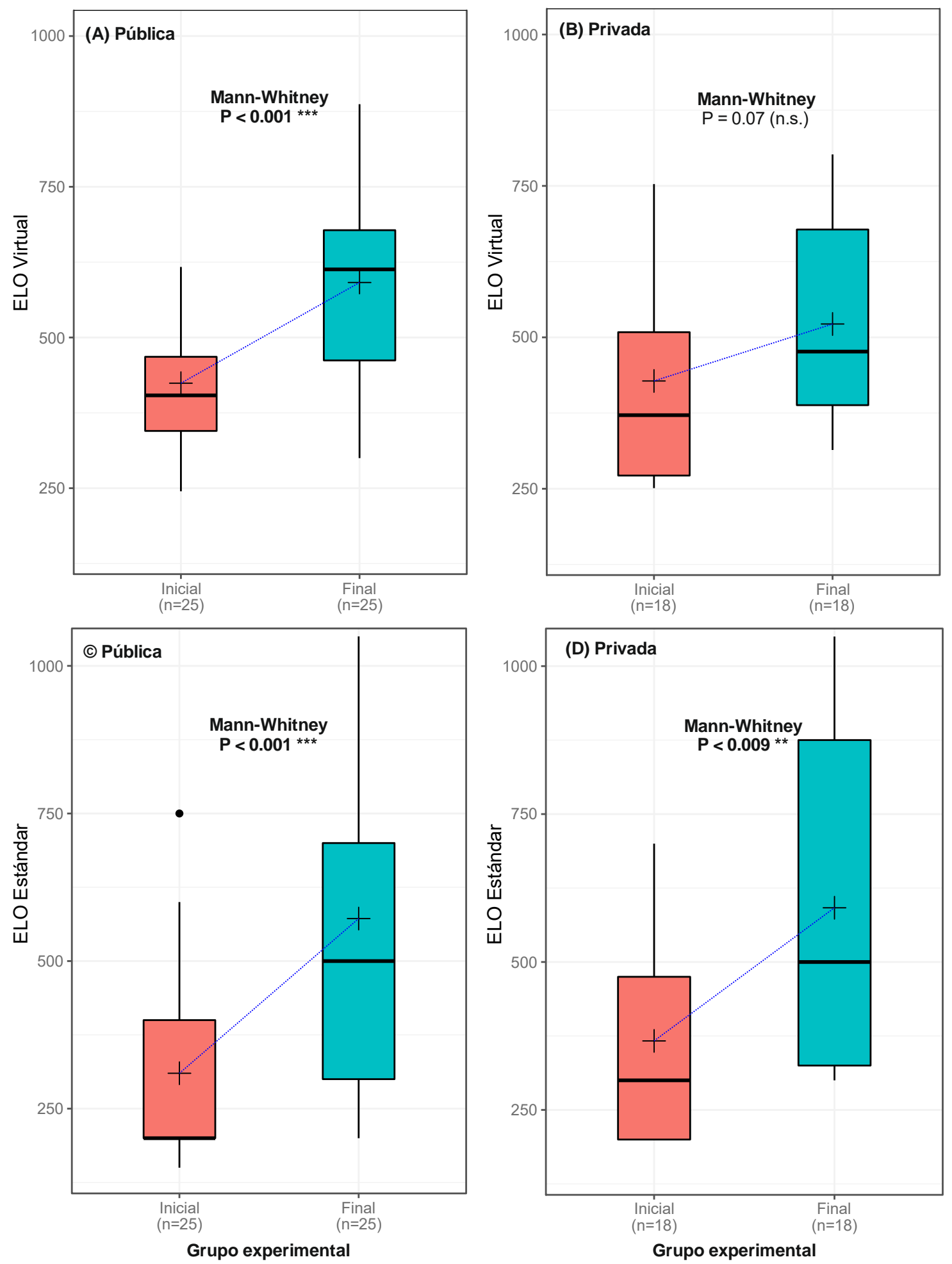

Figura 1. Boxplot de los cambios en el Elo (virtual y estándar) luego de aplicación de ajedrez educativo en dos Instituciones educativa de Puerto Maldonado (Perú). 


\section{Variaciones en el Elo según sexo luego del tratamiento}

Al comparar los incrementos de Elo virtual entre varones y mujeres no se encontraron diferencias significativas $(P>0.05$, Tabla 3A). Aunque se encontró diferencias entre IEs, en la IE pública los varones incrementaron su Elo $32 \%$ más que las mujeres, mientras que en la IE privada fueron las mujeres la que incrementaron su Elo en $77 \%$ más que los varones (Tabla 3A). Sin embargo, los incrementos de Elo-estándar luego del tratamiento fue superior en los varones que en las mujeres en las dos IEs. Aunque solo se encontró diferencias significativas en la IE privada (Mann-Whitney, $P=0.019$ (Tabla 3B).

Tabla 3. Resumen de la comparación en la fuerza ajedrecística inicial (ELO virtual y estándar) según sexo, luego de aplicado el tratamiento en estudiantes del primer grado de secundaria de dos instituciones educativas de Puerto Maldonado (Perú). IE: Institución educativa. SD: Desviación estándar.

\begin{tabular}{lllllll}
\hline Incremento & IE & Sexo & Promedio & SD & Diferencia (\%) & Comparación \\
\hline \multirow{2}{*}{ Pública } & Varones & 183.9 & 112.6 & (+) $32 \%$ & T-student, $\mathrm{t}=1.08$ \\
& & Mujeres & 137.0 & 85.4 & & $P=0.29$
\end{tabular}

(A) Elo-virtual

\begin{tabular}{cccccl} 
Privada & Varones & 67.9 & 86.3 & & Mann-Whitney, $\mathrm{U}=0.37$ \\
& Mujeres & 120.2 & 34.3 & $(+) 77 \%$ & $P=0.37$ \\
& & & & & \\
\multirow{2}{*}{ Pública } & Varones & 278.1 & 249.0 & & $\begin{array}{l}\text { Mann-Whitney, } \mathrm{U}=71.5 \\
\end{array}$ \\
& Mujeres & 233.3 & 147.9 & $(+) 68 \%$ & $P=0.98$
\end{tabular}

(A) Elo-estándar

$\begin{array}{llllll}\text { Privada } & \text { Varones } & 311.1 & 181.6 & (+) 273 \% & \begin{array}{l}\text { Mann-Whitney, } \mathrm{U}=14.5 \\ \end{array} \\ & \text { Mujeres } & 138.9 & 48.6 & & P=0.019\end{array}$

\section{Notas en razonamiento matemático y atención y concentración}

En las dos habilidades evaluadas los resultados obtenidos en las calificaciones en el posttest del grupo experimental son superiores a los alcanzados por el grupo control (Figura 2). El análisis comparativo de las calificaciones de razonamiento lógico matemático entre el grupo control y experimental mostró diferencias después de la aplicación del tratamiento, siendo superior en el grupo experimental en las dos IEs (Figura 2A y 2D). En el grupo control no se observó diferencias entre el pre-test y pos-test (Figura 2B y 2E), asimismo en el grupo experimental no se observó diferencias según sexo (Figura 2C y 2F). 

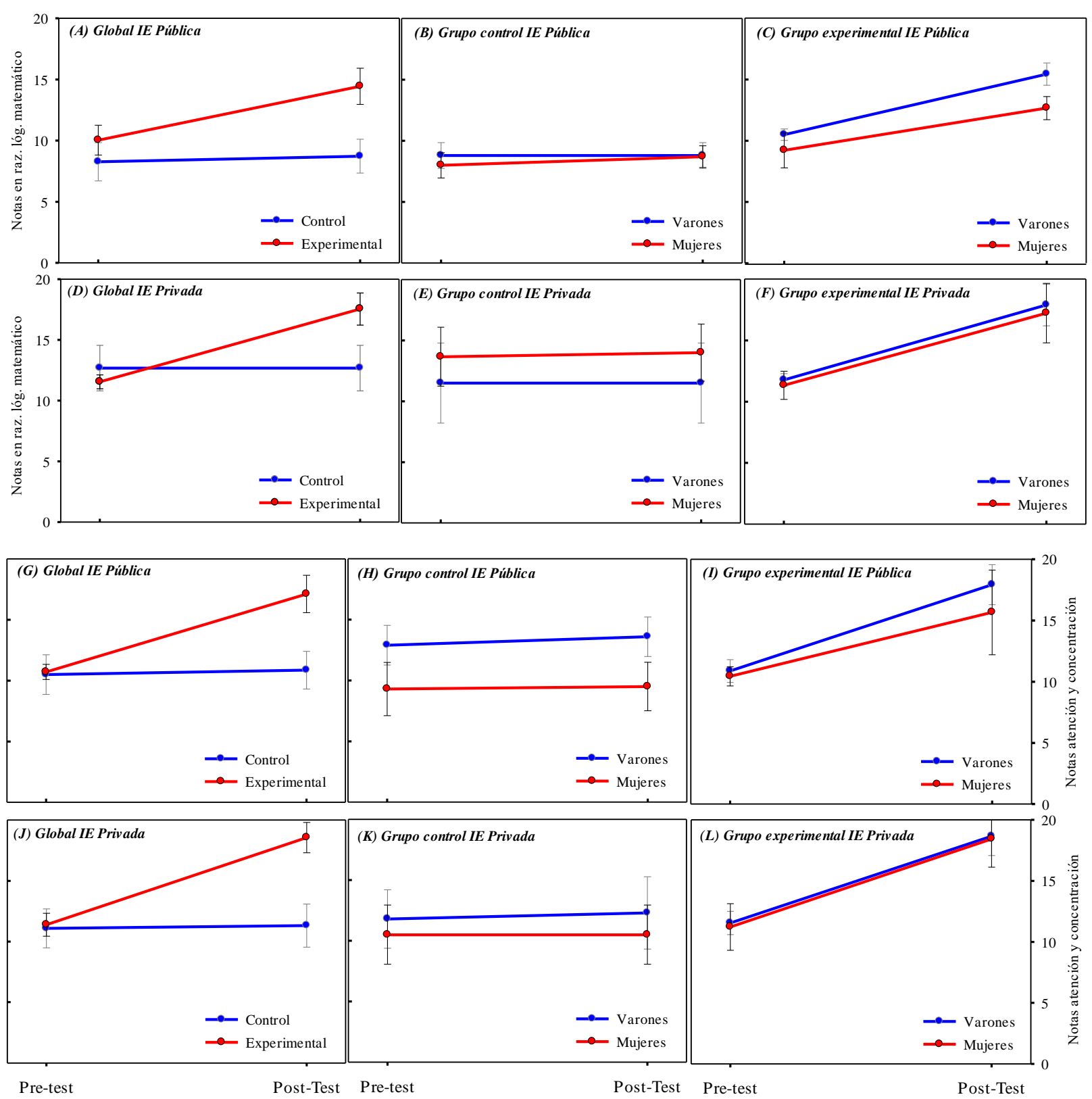

Figura 2. Cambios en el promedio ( \pm error estándar) razonamiento lógico matemático (A-F) y atención y concentración (G-L) por grupo de estudio e institución educativa después del tratamiento.

Por otro lado, el análisis comparativo de las calificaciones de atención y concentración entre el grupo control y experimental mostró diferencias después de la aplicación del tratamiento, siendo superior en el grupo experimental en las dos IEs (Figura $2 \mathrm{G}$ y 2J). En el grupo control no se observó 
diferencias entre el pre-test y post-test (Figura $2 \mathrm{H}$ y $2 \mathrm{~K}$ ). Sin embargo, en el grupo experimental de la IE pública, el incremento en las calificaciones fue superior en los varones (Figura 2I), mientras que en la IE privada no se observó diferencias según sexo (Figura 2L).

\section{Efecto de la aplicación del ajedrez en el desarrollo de razonamiento lógico matemático}

El GLM ajustado para el experimento de estudiantes de primer grado de secundaria en las dos IEs resultó significativo $(P<0.001$, Tabla 4). El porcentaje de variabilidad explicada (Devianza $\mathrm{D}^{2}$ ) en la variable respuesta por el GLM ajustado fue de $73.2 \%$ en la IE pública y $78.6 \%$ en la IE privada. Se encontró un efecto significativo de la aplicación del ajedrez deportivo en el desarrollo de habilidades en razonamiento lógico matemático en estudiantes de la IE pública $\left(X^{2}=56.8, P<\right.$ 0.001 , Tabla 4) y privada $\left(X^{2}=29.1, P<0.001\right)$. Entre grupos (control y experimental) de ambas IEs no encontramos diferencias significativas en la respuesta según sexo $(P>0.05$, Tabla 4$)$.

Tabla 4. Resultados del GLM sobre el efecto del ajedrez en el rendimiento de lógico matemático en estudiantes del primer grado de secundaria de dos instituciones educativas de Puerto Maldonado (Perú).

\begin{tabular}{|c|c|c|c|c|}
\hline \multicolumn{5}{|l|}{ (A) Efectos del modelo } \\
\hline \multirow[t]{2}{*}{ Institución educativa } & \multicolumn{2}{|c|}{ Pública } & \multicolumn{2}{|c|}{ Privada } \\
\hline & $\begin{array}{c}X^{2} \text { razón de } \\
\text { verosimilitud }\end{array}$ & $P$ & $\begin{array}{c}X^{2} \text { razón de } \\
\text { verosimilitud }\end{array}$ & $P$ \\
\hline Grupo (Control-Experimental) & 56.79 & $<0.001 *$ & 29.1 & $<0.001 *$ \\
\hline Sexo (Grupo) & 4.39 & 0.114 & 0 & 0.993 \\
\hline Pretest (Covariable) & 151.7 & $<0.001 *$ & 32.4 & $<0.001 *$ \\
\hline
\end{tabular}

(B) Estimaciones de los parámetros

\begin{tabular}{lcccccc} 
Institución educativa & \multicolumn{3}{c}{ Pública } & \multicolumn{3}{c}{ Privada } \\
& Parámetro $(\beta)$ & $X^{2}$ Wald & $P$ & Parámetro $(\beta)$ & $X^{2}$ Wald & $P$ \\
(Intersección) & 1.692 & 344 & $<0.001^{*}$ & 1.883 & 97.839 & $<0.001^{*}$ \\
Grupo (Tratamiento-Control) & -0.291 & 18.4 & $<0.001^{*}$ & -0.466 & 14.623 & $<0.001^{*}$ \\
Sexo (Control) & -0.021 & 0.1 & 0.775 & -0.014 & 0.012 & 0.912 \\
Sexo (Experimental) & 0.13 & 4.2 & $0.041^{*}$ & 0.006 & 0.003 & 0.958 \\
Pretest (Covariable) & 0.086 & 143.9 & $<0.001^{*}$ & 0.084 & 31.618 & $<0.001 *$ \\
\hline
\end{tabular}


Sin embargo, dentro el grupo experimental de la IE pública se encontró una diferencia significativa según sexo en el desarrollo de habilidades en razonamiento lógico matemático $\left(X^{2}=\right.$ 4.2, $P<0.05$, Tabla 4 y Figura 2I). Esto sugiere que el ajedrez deportivo tuvo un efecto superior en el razonamiento lógico matemático en los varones (+4.94 en promedio) que en las mujeres (+3.44 en promedio) (Figura 3D). Por otro lado, el análisis de las medias marginales mostró que la aplicación del ajedrez incrementó, en promedio, el desarrollo de la habilidad intelectual razonamiento lógico matemático en 3.9 puntos en la IE publica y en 6.9 puntos en la IE privada (Figura 3A,3B).
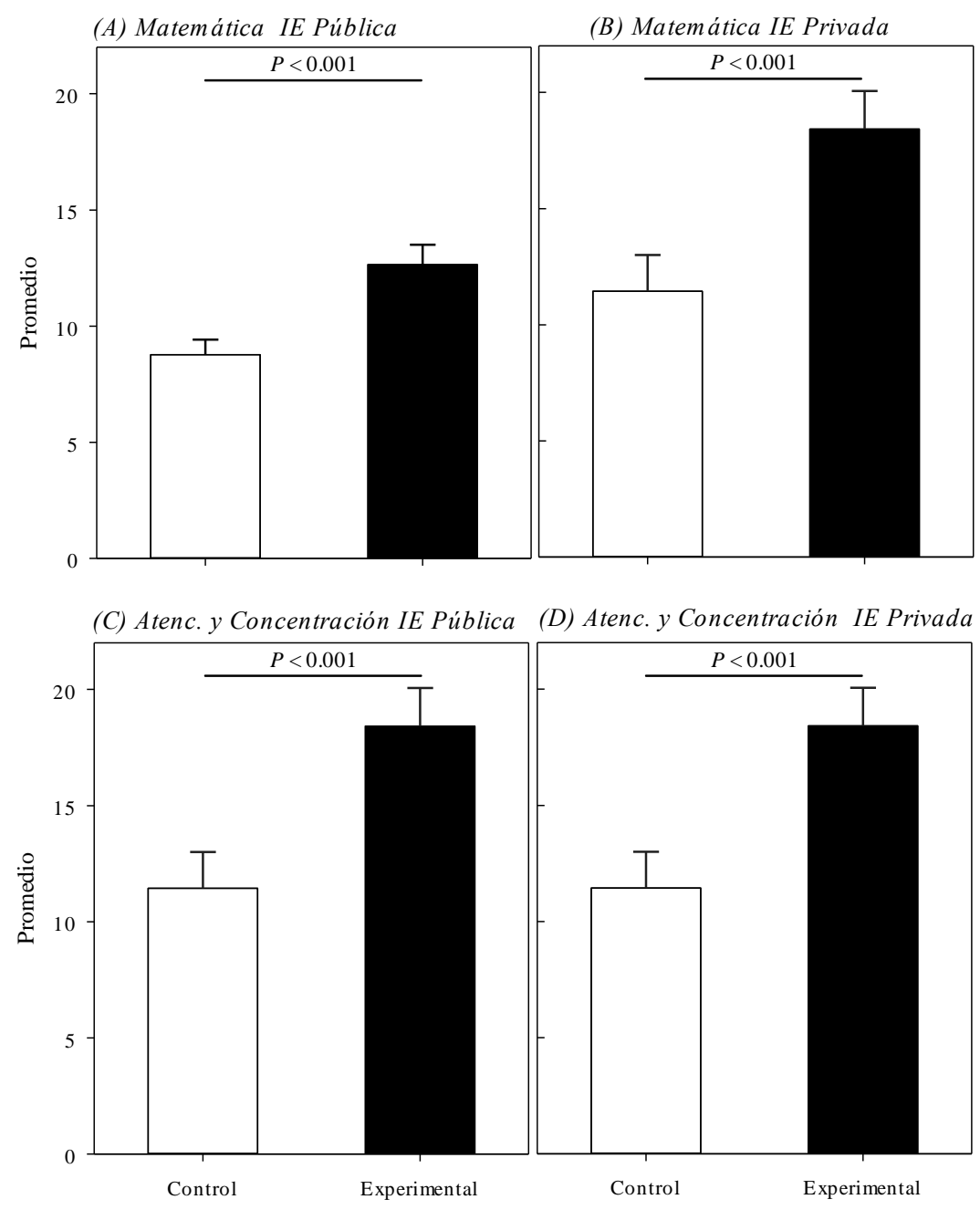

Figura 3. Incremento de las calificaciones en las habilidades de razonamiento lógico matemático (A y B) y atención y concentración ( $\mathrm{C}$ y D) por grupo de estudio e institución educativa después del tratamiento. Las barras representan la media marginal y los bigotes representan a los intervalos confianza de Wald (95\%). 


\section{Efecto de la aplicación del ajedrez deportivo en el desarrollo de la habilidad de atención y concentración}

El GLM ajustado para el experimento de estudiantes de primer grado de secundaria en las dos IEs resultó significativo $(P<0.001$, Tabla 5). El porcentaje de variabilidad explicada en la variable respuesta por el GLM ajustado fue de $75.3 \%$ en la IE pública y $72.7 \%$ en la IE privada. Se encontró un efecto significativo de la aplicación del ajedrez deportivo en el desarrollo de la habilidad intelectual razonamiento lógico matemático en los estudiantes del primer grado de la IE pública $\left(X^{2}=56.8, P<0.001\right.$, Tabla 5) y la IE privada $\left(X^{2}=36.4, P<0.001\right.$, Tabla 5). Entre grupos (control y experimental) y dentro de los grupos de ambas IEs no encontramos diferencias significativas en la respuesta según sexo $(P>0.05$, Tabla 5). Por otro lado, el análisis de las medias marginales mostró que la aplicación del ajedrez incrementó, en promedio, el desarrollo de la habilidad intelectual de atención y concentración en 6.2 puntos en la IE pública y en 7.1 puntos en la IE privada (ver arriba, Figura 3C y 3D).

Tabla 5. Resultados del GLM sobre el efecto del ajedrez en atención y concentración en estudiantes del primer grado de secundaria de dos instituciones educativas de Puerto Maldonado (Perú).

\begin{tabular}{lcccc}
\hline (A) Efectos del modelo & \multicolumn{3}{c}{ Privada } \\
\hline Institución educativa & \multicolumn{2}{c}{ Pública } & \multicolumn{2}{c}{$X^{2}$ razón de } \\
& $\begin{array}{c}X^{2} \text { razón de } \\
\text { verosimilitud }\end{array}$ & $P$ & 36.4 & $<$ \\
verosimilitud & \\
Grupo (Control-Experimental) & 58.2 & $<0.001$ & 0.2 & 0.916 \\
Sexo (Grupo) & 2.3 & 0.332 & 31.6 & $<0.001$ \\
Pretest (Covariable) & 61.4 & $<0.001$ &
\end{tabular}

(B) Estimaciones de los parámetros

\begin{tabular}{lccccccc} 
Institución educativa & \multicolumn{3}{c}{ Pública } & \multicolumn{3}{c}{ Privada } \\
& Parámetro $(\beta)$ & $X^{2}$ Wald & $P$ & Parámetro $(\beta)$ & $X^{2}$ Wald & $P$ \\
(Intersección) & 1.814 & 163.32 & $<0.001$ & 1.845 & 73.4 & $<0.001$ \\
Grupo (Tratamiento-Control) & -0.471 & 29.12 & $<0.001$ & -0.533 & 20.3 & $<0.001$ \\
Sexo (Control) & 0.095 & 1.06 & 0.303 & 0.053 & 0.2 & 0.677 \\
Sexo (Experimental) & 0.09 & 1.24 & 0.265 & -0.004 & 0 & 0.97 \\
Pretest (Covariable) & 0.089 & 56.11 & $<0.001$ & 0.093 & 29.3 & $<0.001$ \\
\hline
\end{tabular}




\section{Discusión}

Se confirmó la hipótesis del presente estudio, según la cual los incrementos en las puntuaciones de razonamiento matemático y atención-concentración sería significativamente superior en el grupo que practicó el ajedrez (experimental) que en el grupo control. Nuestra hipótesis es soportada por otros estudios que demuestran que las lecciones de ajedrez pueden ser efectivas para mejorar las habilidades en matemáticas y concentración (Costello, 2013; Gao et al., 2019; H. García y Blanch, 2016; Grau-Pérez y Moreira, 2017; Jankovic y Novak, 2019; Rosholm et al., 2017; Sala, et al. 2016), aunque podría ser más efectiva si la intervención se complementa con un entrenamiento en el hogar (Sala, Gorini, y Pravettoni, 2015) y se convierte en una práctica constante en las instituciones educativas.

En las dos IEs (pública y privada) las puntuaciones alcanzadas por los estudiantes del grupo experimental en las habilidades en razonamiento matemático y atención-control son significativamente superiores a los alcanzados en el grupo control (Figura 3). Esto comprobaría la eficacia de la implementación del ajedrez deportivo en los estudiantes para el desarrollo de estas habilidades. Sala et al. (2015) sugiere que incluso una práctica breve de ajedrez en niños puede ser una herramienta útil para mejorar sus habilidades matemáticas.

En consecuencia, las mejoras en la habilidades cognitivas evaluadas en el presente estudio estarían relacionadas a que la práctica del ajedrez ejercería una influencia positiva en la capacidad de concentración, un proceso importante al momento de estudiar (Kovacic, 2012). Además, practicar o jugar regularmente al ajedrez está vinculado a otras habilidades que son importantes en la resolución de problemas matemáticos, alto niveles de atención y concentración (Trinchero, 2013; Trinchero y Sala, 2016), y por lo tanto influencia positivamente el aprendizaje de los estudiantes. Según Sala et al. (2015) el ajedrez es por naturaleza un deporte que fuerza a los jugadores a usar sus habilidades en cálculo de variantes (simples y complejas) y ejercicios nemotécnicos (recodar aperturas, finales, etc.). Martínez-Artero y Nortes (2015) y Mesa (2016) sugieren que el ajedrez en las escuelas funcionaria como una herramienta pedagógica y un recurso didáctico incuestionable que favorecería el aprendizaje autónomo de los estudiantes.

Por otro lado, a diferencias de los resultados de (Scholz et al., 2008), nosotros si encontramos evidencia de efecto del ajedrez en la atención y concentración en los estudiantes de durante el periodo de estudio, a pesar del poco tiempo de intervención. Resultados similares de la 
contribución de uso de ajedrez en la atención de estudiantes peruanos fueron reportados por Reluz (2019) en estudiantes de 5to grado de primaria de una institución pública de Chiclayo.

El rendimiento de los estudiantes de la IE de gestión privada fue superior a la de gestión pública, similares resultados fueron reportados por estudiantes de secundaria en el Perú (Bazán, Espinoza, y Farro, 2002). Esta diferencia también fue reportada por la ECE-2018 en el Perú (MINEDU, 2018b), en la cual las IE privadas obtuvieron un mayor porcentaje satisfactorio en la evaluación de matemáticas que las IE públicas, $24 \%$ y $12 \%$, respectivamente.

Sobre el rendimiento según sexo, en el presentes estudio encontramos un mayor rendimiento en los varones en las mujeres, aunque solo significativo para la IE pública. Estos resultados coinciden con otros reportados sobre el rendimiento académico de los estudiantes según sexo en el Perú (Bazán et al., 2002; OECD, 2019; Bos, Ganimian, y Vegas, 2014) y Latinoamérica (Bos et al., 2014; González, 2003; León y Salazar, 2014). Según Bazán et al. (2002) las diferencias en el rendimiento de matemáticas se incrementan en el nivel secundario, en comparación con la primaria, lo cual coincide con los resultados del estudio. Considerando las evaluaciones PISA, en Latinoamérica los varones se desempeñan mejor que las mujeres en matemáticas y en un análisis global los varones superan a las mujeres en las evaluaciones de matemáticas del PISA, mientras que las mujeres se desempeñan mejor que los varones en lectura (Bos et al., 2014). Diversos estudios sugieren que esta esta diferencia según sexo en las matemáticas podría estar relacionado a factores biológicos, metodología de evaluación y que los sistemas educativos no consideran que los niños y niñas suelen procesar y aprender matemáticas de diferente manera (Bos et al., 2014; Geist y King, 2005; León y Salazar, 2014). Aunque York y Clark (2007) sugieren que no hay suficiente evidencia de que las diferencias entre niños y niñas sea debido a factores genéticos, $\mathrm{y}$ que podría estar relacionado a otros factores sociales, como por ejemplo debido a la disparidad de oportunidades.

Por otro lado, considerando los bajos rendimiento académicos en Madre de Dios y en el Perú, el ajedrez como deporte debería utilizarse como una herramienta pedagógica. Sin embargo, los objetivos de este programa deberían estar claros antes de iniciar, es decir no solo se debería practicar con la finalidad de obtener trofeos o campeonatos, sino como una herramienta pedagógica transversal al aprendizaje que ayude en el rendimiento académico y actitudinal (Mesa, 2016). Se debería considerar experiencias de implementación de otros países en el currículo escolar, por ejemplo España, Italia, Reino Unido y Alemania establecieron la enseñanza de ajedrez en las 
escuelas como un componente opcional para los estudiantes (Sala y Gobet, 2016). Por lo tanto se debería promover la práctica de ajedrez en la educación primaria y secundaria (Reluz, 2019).

\section{Limitaciones del estudio y recomendaciones para estudios futuros}

El presente estudio tiene fortalezas como la experiencia en la enseñanza de ajedrez de los instructores y el uso de un grupo control. Sin embargo, el estudio tiene limitaciones que vale la pena mencionar, como asignación aleatoria de los grupos, reducido muestra de estudiantes e IE. Idealmente, se prefiere un experimento aleatorio para determinar el efecto del ajedrez en el rendimiento de los estudiantes (Rosholm et al., 2017). Sala y Gobet (2016) sugieren que un diseño ideal para este tipo de experimento debería incluir un pretest y postest con un grupo tratamiento y dos grupos control (activo y pasivo), una asignación aleatoria de los grupos. Por lo tanto, diseñamos el presente estudio como un estudio piloto, para demostración sobre los posibles efectos positivos de la enseñanza de ajedrez en estudiante de la Amazonia peruana.

\section{Conclusión}

Considerando los resultados del estudio se concluye que la implementación de un programa ajedrez deportivo influye en el desarrollo de las habilidades intelectuales de (1) razonamiento lógico matemático y (2) atención y concentración. Por otro lado, la intervención en el grupo experimental influyó significativamente en el incremento del nivel ajedrecístico (Elo) de los estudiantes, por lo tanto, el tratamiento aplicado fue el adecuado.

Nuestros resultados aportan información relevante y son el sustento para que las instituciones educativas pueden desarrollar estrategias para fomentar y promover el ajedrez como una herramienta pedagógica e incluir al ajedrez en el currículo escolar peruano. Mas aun cuando a nivel internacional los sistemas educativos vienen modernizando los métodos de enseñanza y algunos países (Canadá, Reino Unido, España, Turquía, Alemania, Italia, entre otros) consideran al ajedrez como adecuado dentro de sus programas de matemáticas.

\section{Referencias}

Aciego, R., García, L., y Betancort, M. (2016). Efectos del método de entrenamiento en ajedrez, entrenamiento táctico versus formación integral, en las competencias cognitivas y sociopersonales de los escolares. Universitas Psychologica, 15(1), 165-176. 
https://doi.org/10.11144/Javeriana.upsy15-1.emea

Avalos, B., Morales, I., Jimenez, W., y Vaca, K. (2019). Influencia del ajedrez en el desarrollo integral del hombre. Universidad, Ciencia y Tecnología, 02, 100-108. https://www.uctunexpo.autanabooks.com/index.php/uct/article/view/226/334

Bazán, J., Espinoza, G., y Farro, C. (2002). Rendimiento y actitudes hacia la matemática en el sistema escolar peruano. Lima: Ministerio de Educación, Peru. http://argos.pucp.edu.pe/ jlbazan/download/13c.pdf

Benítez, J., y Roca, A. (2018). Las matemáticas escondidas en la puntuación Elo del ajedrez Introducción Entra en juego la distribución normal. La Gaceta de la Real Sociedad $\begin{array}{lll}\text { Matemática Española, } & \text { 517-525. }\end{array}$ https://dialnet.unirioja.es/servlet/articulo?codigo=6930007

Bos, M., Ganimian, A., y Vegas, E. (2014). América Latina en el PISA 2012. Brief\#5: ¿Como se desempeñan los varones y las mujeres? http://hdl.handle.net/123456789/2559

Costello, P. J. M. (2013). The gymnasium of the mind: Teaching chess in early childhood. Early Child Development and Care, 183(8), 1133-1146. https://doi.org/10.1080/03004430.2012.759568

Dugard, P., y Todman, J. (1995). Analysis of Pre-test-Post-test Control Group Designs in Educational Research. Educational Psychology, 15(2), 181-198. https://doi.org/10.1080/0144341950150207

Fernández, A., Ortega, J., y Oliveras, M. (2018). El ajedrez en la enseñanza-aprendizaje de áreas científicomatemáticas, una revisión bibliográfica en el contexto español. Revista Anales, 1(376), 57-65. https://doi.org/10.29166/anales.v1i376.1763

Fernández, J., y Sánchez, M. (2011). Canto al ajedrez: enseñar ajedrez con canciones. Tendencias pedagógicas, 18, 269-322. http://dialnet.unirioja.es/servlet/articulo?codigo=3780676

Gao, Q., Chen, W., Wang, Z., y Lin, D. (2019). Secret of the masters: Young chess players show advanced visual perspective taking. Frontiers in Psychology, 10, 2407. https://doi.org/10.3389/fpsyg.2019.02407

García, A. (2018). Ajedrez en el proceso educativo de los adolescentes (Tesis pregrado). Universidad Nacional de Tumbes, Piura, Perú. Recuperado de http://repositorio.untumbes.edu.pe/handle/UNITUMBES/513

García, H., y Blanch, Á. (2016). Tecnochess. Una propuesta didáctica innovadora para trabajar las 
competencias lingüística, tecnológica y matemática mediante el juego del ajedrez y las TIC. Revista Interuniversitaria de Investigación en Tecnología Educativa, 1, 39-51. https://doi.org/10.6018/riite/2016/263991

Geist, E. A, y King, M. (2005). Different, Not Better: Gender Differences in Mathematics Learning and Achievement. Journal of Instructional Psychology, 35, 43-52. https://www.questia.com/library/journal/1G1-178218787/

González, R. (2003). Diferencias de género en el desempeño matemático de estudiantes de secundaria. $\quad$ Educación matemática, $129-162$. https://www.redalyc.org/articulo.oa?id=40515206

Grau-Pérez, G., y Moreira, K. (2017). A study of the influence of chess on the Executive Functions in school-aged children / Estudio del impacto del ajedrez sobre las Funciones Ejecutivas en niños de edad escolar. Estudios de Psicologia, 38(2), 473-494. https://doi.org/10.1080/02109395.2017.1295578

Hammer, Ø., Harper, D., y Ryan, D. (2001). Past: Paleontological Statistics Software Package for Education and Data Analysis. Palaeontologia Electronica, 4(4), 5-7. https://palaeoelectronica.org/2001_1/past/past.pdf

Jankovic, A., y Novak, I. (2019). Chess as a Powerful Educational Tool for Successful People. EconStor. Recuperado de http://hdl.handle.net/10419/196101

Johnson, T. R. (2016). Violation of the homogeneity of regression slopes assumption in ANCOVA for two-group pre-post designs: Tutorial on a modified Johnson-Neyman procedure. The Quantitative Methods for Psychology, 12(3), 253-263. https://doi.org/10.20982/tqmp.12.3.p253

Kovacic, D. (2012). Chess in Schools. A Good Move. PSIENCIA Revista Latinoamericana de Ciencia Psicológica, 4(1), 29-41. https://doi.org/10.5872/psiencia.v4i1.87

Lara-Piña, R., Vargas, E., Erosa, E., Mestas, L., y Rincón, S. (2018). On the relationship of working memory and chess in a psychology major freshmen sample: exploratory study. Revista Electrónica de Psicología, 8(15), 12-17. https://www.zaragoza.unam.mx/wpcontent/Portal2015/publicaciones/revistas/rev_elec_psico/REP_15.pdf

León, V., y Salazar, A. (2014). Diferencias de género en matemática y lenguaje en alumnos de colegios adventistas en el sistema de medición de la calidad de la Educación (SIMCE) en Chile. Apuntes Universitarios, 4(2), 81-106. Recuperado de 
http://www.redalyc.org/articulo.oa?id=467646129005

Lotero, F., y Cano, O. (2017). Tactical component in chess performance: Proposal of a method of teaching and training in childhood. Revista de Educación Física, 6(4), 80-159. https://revistas.udea.edu.co/index.php/viref/article/view/329476

Martínez-Artero, R., y Nortes, A. (2015). El ajedrez como recurso didáctico en la enseñanzaaprendizaje de las Matemáticas. Números, (89), 9-31. http://www.sinewton.org/numeros/numeros/89/Articulos_01.pdf

Mesa, J. (2016). El ajedrez como herramienta pedagógica que favorece el aprendizaje autónomo (Tesis de pregrado). Universidad Abierta y a Distancia - UNAD, Antioquia, Colombia. https://repository.unad.edu.co/handle/10596/11696

MINEDU. (2015). Madre de Dios: ¿cómo vamos en educación?. Unidad de estadistica 2015. Estadistica de la calidad educativa, Lima, Perú. https://doi.org/http://repositorio.minedu.gob.pe/handle/123456789/4713

MINEDU. (2018a). ¿Qué aprendizajes logran nuestros estudiantes? Lima, Perú: Ministerio de Educación. http://repositorio.minedu.gob.pe/handle/MINEDU/6588

MINEDU. (2018b). Evaluación Censal de Estudiantes 2018. Lima, Perú: Ministerio de Educación. https://doi.org/http://repositorio.minedu.gob.pe/handle/123456789/3892

Muelle, L. (2020). Socioeconomic and contextual factors associated with low academic performance of peruvian students in PISA 2015. Apuntes, 47(86), 111-146. https://doi.org/10.21678/apuntes.86.943

Nakao, M. (2019). Special series on "effects of board games on health education and promotion" board games as a promising tool for health promotion: A review of recent literature. BioPsychoSocial Medicine, 13(1), 3-9. https://doi.org/10.1186/s13030-019-0146-3

OECD. (2019). PISA 2018 insights and interpretations. En OECD Publishing. https://doi.org/https://doi.org/10.1787/19963777

Poston, D. I., y Vandenkieboom, K. K. (2019). The Effect of Chess on Standardized Test Score Gains. SAGE Open, 9(3). https://doi.org/10.1177/2158244019870787

R Core Team. (2017). R: A language and environment for statistical computing. http://www.Rproject.org/.

Ramos, L., Arán-Filippetti, V., y Krumm, G. (2017). Funciones ejecutivas y práctica de ajedrez: un estudio en niños escolarizados. Psicogente, 21(39), 25-34. 
https://doi.org/10.17081/psico.21.39.2794

Reluz, J. I. (2019). Uso del ajedrez para mejorar los niveles atencionales de estudiantes de primaria en una institución educativa pública. Educare et Comunicare, 7(1), 41-50. https://doi.org/10.35383/educare.v7i1.224

Rosholm, M., Mikkelsen, M. B., y Gumede, K. (2017). Your move: The effect of chess on $\begin{array}{lllll}\text { mathematics } & \text { test } & \text { scores. } & \text { PLoS } & \text { ONE, }\end{array}$ https://doi.org/10.1371/journal.pone.0177257

Sala, G., y Gobet, F. (2016). Do the benefits of chess instruction transfer to academic and cognitive skills? A meta-analysis. Educational Research Review, 18, 46-57. https://doi.org/10.1016/j.edurev.2016.02.002

Sala, G., Gobet, F., Trinchero, R., y Ventura, S. (2016). Does chess instruction enhance mathematical ability in children? A three-group design to control for placebo effects. Proceedings of the 38th Annual Meeting of the Cognitive Science Society. http://hdl.handle.net/2318/1638111

Sala, G., Gorini, A., y Pravettoni, G. (2015). Mathematical Problem-Solving Abilities and Chess: An Experimental Study on Young Pupils. SAGE Open, 5(3), 1-9. https://doi.org/10.1177/2158244015596050

Salinas, A. (2015). El juego de ajedrez y el desarrollo de las habilidades matemáticas en los estudiantes de educación secundaria básica (Universidad Nacional del Santa). https://doi.org/http://repositorio.uns.edu.pe/handle/UNS/2820

Scholz, M., Niesch, H., Steffen, O., Ernst, B., Loeffler, Witruk, M., y E. Schwarz, H. (2008). Impact of chess training on mathematics performance and concentration ability of children with learning disabilities. International Journal of Special Education, 23(3), 138-148. http://www.internationalsped.com/documents/Scholz\%20et\%20al\%20Chess.doc

Sotelo, N., Sotelo, C., Matalinares, M. L., Arenas-Iparraguirre, C., y Caycho, T. (2014). Efectos de un programa experimental interactivo para mejorar la comprensión lectora en una población rural de 5. ${ }^{\circ}$ grado de educación primaria. Revista de Investigación en Psicología, 15(1), 93107. https://doi.org/10.15381/rinvp.v15i1.3671

Storey, K. (2000). Teaching Beginning Chess Skills to Students With Disabilities. Preventing School Failure: Alternative Education for Children and Youth, 44(2), 45-49. https://doi.org/10.1080/10459880009599782 
Surco-Huacachi, O. (2020). Aprendiendo Ajedrez: Guia de aprendizahe nivel iniciación y básico. Puerto Maldonado: Amazon.

Trinchero, R. (2013). Can chess training improve Pisa scores in mathematics. An Experiment in Italian Primary School. https://aperto.unito.it/retrieve/handle/2318/142194/23444/

Trinchero, R., y Sala, G. (2016). Chess training and mathematical problem-solving: The role of teaching heuristics in transfer of learning. Eurasia Journal of Mathematics, Science and Technology Education, 12(3), 655-668. https://doi.org/10.12973/eurasia.2016.1255a

Wickham, H. (2009). ggplot2 Elegant Graphics for Data Analysis. En Media (Vol. 35). https://doi.org/10.1007/978-0-387-98141-3

York, R., y Clark, B. (2007). Gender and mathematical ability: The toll of biological determinism. Monthly Review. https://doi.org/10.14452/MR-059-06-2007-10_2 